\title{
IMIII Assessment of HBV Preventive Services in a Medically Underserved Asian and Pacific Islander Population Using Provider and Patient Data
}

\author{
Nizar A. Mukhtar, $M D^{7}$, Brian C. Toy, $M D^{7}$, Blaire E. Burman, $M D^{7}$, Albert Yu, MD², \\ Alice $\mathrm{Hm}$ Chen, $M D^{7}$, Peter Berman, $\mathrm{MD}^{2}$, Tung Nguyen, $\mathrm{MD}^{7}$, Daniel Chan, $\mathrm{MD}^{4}$, \\ Hali Hammer, MD ${ }^{2}$, Charles E. McCulloch, $P h D^{5}$, and Mandana Khalili, MD ${ }^{7,3,6}$
}

'Department of Medicine, University of California, San Francisco, San Francisco, CA, USA; ${ }^{2}$ Department of Family and Community Medicine, University of California, San Francisco, San Francisco, CA, USA; ${ }^{3}$ Liver Center, University of California, San Francisco, San Francisco, CA, USA; ${ }^{4}$ North East Medical Services, San Francisco, CA, USA; ${ }^{5}$ Department of Epidemiology and Biostatistics, University of California, San Francisco, San Francisco, CA, USA; 'San Francisco General Hospital, University of California, San Francisco, San Francisco, CA, USA.

BACKGROUND: Hepatitis B (HBV) represents a significant health disparity among medically underserved Asian and Hawaiian/Pacific Islander (API) populations. Studies evaluating adherence to HBV screening and vaccination guidelines in this population are limited.

OBJECTIVE: The purpose of this study was to evaluate HBV screening and vaccination practices using both provider self-report and patient records.

DESIGN: Medical records for 20,574 API adults were reviewed retrospectively and primary care providers were surveyed to evaluate rates and adherence to HBV screening and vaccination guidelines.

PARTICIPANTS: The study included primary care providers and their adult API patients in the San Francisco safety-net healthcare system.

MAIN MEASURES: Patient, practice, and provider factors, as well as HBV screening and vaccination practices, were assessed using provider survey constructs and patient laboratory and clinical data. Generalized linear mixed models and multivariate logistic regression analyses were used to identify factors associated with recommended HBV screening and vaccination.

KEY RESULTS: The mean age of patients was 52 years, and $63.4 \%$ of patients were female. Only $61.5 \%$ underwent HBV testing, and $47.4 \%$ of HBV-susceptible patients were vaccinated. Of 148 (44.8\%) responding providers, most were knowledgeable and had a favorable attitude towards screening, but $43.2 \%$ were unfamiliar with HBV guidelines. HBV screening was positively associated with favorable provider attitude score (OR per unit 1.80, $95 \%$ CI 1.18-2.74) and negatively associated with female patient sex (OR 0.82, $95 \%$ CI 0.73-0.92), a higher number of clinic patients per week (OR per 20 patients 0.46, $95 \%$ CI 0.28-0.76), and provider barrier score (OR per unit $0.45,95 \%$ CI $0.24-0.87$ ). HBV vaccination was negatively associated with provider barrier score (OR per unit 0.48, $95 \%$ CI 0.25-0.91).

Received November 26, 2013

Revised May 22, 2014

Accepted September 24, 2014

Published online October 17, 2014
CONCLUSIONS: Rates of HBV screening and vaccination of API patients in this safety-net system are suboptimal, and provider factors play a significant role. Efforts to cultivate positive attitudes among providers and expand healthcare system resources to reduce provider barriers to HBV care are warranted.

KEY WORDS: HBV screening; HBV vaccination; HBV prevention; Safety net; Asians.

J Gen Intern Med 30(1):68-74

DOI: $10.1007 / \mathrm{s} 11606-014-3057-9$

(c) Society of General Internal Medicine 2014

\section{INTRODUCTION}

Chronic hepatitis B virus (HBV) infection represents a significant public health burden in the United States, affecting more than 1.25 million individuals. ${ }^{1}$ Asian and Hawaiian/Pacific Islanders (API) represent a particularly high-risk population, with reported prevalence rates of 10-15\%, and are estimated to account for over $40 \%$ of chronic HBV cases in the U.S. ${ }^{2}$ $\mathrm{HBV}$ is associated with increased morbidity and mortality; $15-40 \%$ of patients will develop cirrhosis, liver failure, or hepatocellular carcinoma (HCC). ${ }^{3}$ The recent U.S. Institute of Medicine report highlights the need for increasing efforts in the United States to screen for, prevent, and treat HBV infection. ${ }^{4}$ To meet this goal, it is imperative that we have a better understanding of the complex interplay between patient, provider, and health system factors limiting preventive care and progress towards HBV eradication.

The 2009 American Association for the Study of Liver Diseases (AASLD) guidelines ${ }^{1}$ on HBV screening and management incorporate the Centers for Disease Control and Prevention $(\mathrm{CDC})^{5}$ recommendations, but also identify additional HBV risk groups for screening. Both CDC and AASLD recommend screening immigrants from regions of intermediate to high prevalence of $\mathrm{HBV}$, United States-born persons 
whose parents are from regions of high HBV endemicity, and persons with high-risk behaviors, among others. ${ }^{1,5}$ While the 2004 USPSTF statement recommended against HBV screening in asymptomatic non-pregnant adults, its recently published draft statement is now in line with screening high-risk groups as identified by AASLD guidelines. ${ }^{6}$ The AASLD recommends testing with both $\mathrm{HBsAg}$ and anti-HBs to screen for $\mathrm{HBV}$ and identify those eligible for vaccination.

The San Francisco Bay Area offers a unique setting for evaluation of HBV screening and management practices, given its ethnic diversity and large API community with an estimated HBV prevalence of $8.9 \%{ }^{7}$ Further, given that up to $30 \%$ of patients in the San Francisco safety-net healthcare system self-identify as API, ${ }^{8}$ providers functioning within this system have high exposure to an at-risk population, and an understanding of practice patterns in this setting provides meaningful insight into the factors driving HBV-related healthcare disparities.

Although the procedure is cost-effective, ${ }^{9-12}$ rates of HBV screening and vaccination among at-risk populations are inadequate, and adherence to guidelines for screening of at-risk persons is not well known. Limited studies based largely on provider self-report suggest suboptimal HBV screening and vaccination among API patients, with rates as low as 35$65 \% \cdot{ }^{13-17}$ However, provider self-reporting of clinical practice and preventive services is of limited reliability. ${ }^{18}$ To our knowledge, there are no reports evaluating HBV screening and vaccination practices by both patient record review and provider self-report. Therefore, we aimed to perform a comprehensive assessment of HBV screening and vaccination practices within the San Francisco safety-net healthcare system using provider, practice, and patient data. Specifically, our aims were to 1) assess provider knowledge, attitudes, and barriers towards HBV screening and vaccination, and 2) evaluate rates of and adherence to $\mathrm{HBV}$ screening and vaccination guidelines.

\section{METHODS}

\section{Provider Survey}

Providers were surveyed at primary care clinics within the San Francisco Community Health Network (CHN) between July and December of 2010. The CHN is a safety-net system that provides care to over 150,000 patients per year, including uninsured residents in San Francisco. ${ }^{8}$ This system comprises the San Francisco Department of Public Health (SFDPH), which includes a network of 15 primary care clinics and the San Francisco General Hospital (SFGH), an academic medical center that serves as an acute care and referral facility, as well as 11 primary care clinics affiliated with the San Francisco Community Clinic Consortium.

There are approximately 330 full- and part-time primary care providers (including attending physicians, residents, and nurse practitioners) within the system. The survey was sent to these providers by mail or electronic mail, and subsequent mailings to non-respondents were conducted after four weeks. Provider responses were de-identified for data analysis.

The survey instrument was developed by study investigators with input from experts in primary care, hepatology, and survey design, using a previously published related survey. ${ }^{13}$ Content domains included provider and practice characteristics, HBV screening, vaccination, and management practices; familiarity with HBV management guidelines; HCC screening practices, including modalities used; and provider attitudes about and perceived barriers towards HBV care. The survey was pilot-tested with 20 physicians and then revised.

\section{Review of Electronic Medical Records}

At the time of survey administration, a review was conducted of electronic medical records (EMRs) for patients within the primary care electronic disease registry of the San Francisco safety net who were documented as being Asian or Hawaiian/ Pacific Islander. De-identified patient data were extracted, including patient demographics (age, sex, race/ethnicity, insurance status, clinic location), hepatitis screening and vaccination practices, and laboratory values. The electronic disease registry includes HBV serologic testing data collected since 1997. This study was approved by the UCSF Committee on Human Research.

\section{Data Analysis}

Provider-level Analysis. Data were summarized using mean \pm $\mathrm{SD}$, and characteristics of the providers and their practices are described using frequencies in Tables 1 and 2. In order to assess overall provider knowledge, favorable attitudes, and barriers to $\mathrm{HBV}$ care, composite scores were calculated from responses to the questions designed to assess these factors. Specifically, the knowledge score was computed as the number of correct responses to 11 questions assessing knowledge (1 for correct, 0 for incorrect; max score 11). The attitude score was determined by summing the numerical codes assigned to responses to the seven questions designed to assess attitudes ( 1 for "agree" response, 0.5 for "unsure" response, and 0 for "disagree" response; max score 8). The barrier score was also determined by summing the numerical codes for the nine questions regarding perceived barriers in their practice (1 for "agree" response, 0.5 for "unsure" response, and 0 for "disagree" response; max score 9). Higher composite scores represent higher knowledge, favorable attitude, and greater barriers to HBV care. Table 3 reports percentage correct for each question and mean \pm SD for the composite scores.

Patient-Level Analysis. The primary outcome variables were both binary and measured (and analyzed) at the patient level: 1) adherence to recommended HBV screening guidelines, 
defined as use of both HBsAg and anti-HBs tests, and 2) vaccination of HBV-susceptible patients (HBsAg-negative/ anti-HBs-negative). Vaccination was defined as documentation of receipt of at least one dose of hepatitis vaccine in the primary care electronic disease registry. Provider factors associated with recommended HBV screening and vaccination practices were summarized at the practice level to retain confidentiality by using percentage for binary predictors (e.g., percent male providers) or median for numeric variables (e.g., median provider age). Individual patient-level data were linked with summarized provider data by medical home practice to conduct generalized linear mixed-model logistic regression on individual patients and summarized provider factors associated with recommended HBV screening and vaccination practices (Table 4). This approach accommodates predictors measured at either the practice or patient level and clustering of patients within a practice. Predictors used in the multivariable models included those identified as important a priori (patient age and sex; provider age, sex, and Asian race; provider knowledge, attitude and barrier scores; and provider patient load), as well as those determined by univariate analysis to be statistically significant, with a $p$ value $<0.05$ (twosided). All analyses were performed using Stata version 12.0 (StataCorp, College Station, TX).

\section{RESULTS}

\section{HBV Screening and Vaccination Practices Based on Provider Self-Report}

A total of $148(44.8 \%)$ providers responded to the survey. The proportion of providers from clinics with high HBV patient loads who responded was similar to those who did not respond ( $45.0 \%$ vs. $44.0 \%$, respectively, $p=0.42$ ). The majority of providers were female, self-identified as Caucasian, were MDs, and were in clinical practice for 10 years or less (Table 1). Nearly half served a patient panel comprising more than $25 \%$ Asians, and $39.9 \%$ reported a patient panel with greater than $50 \%$ of patients having limited English proficiency.

One-third of providers reported HBV screening rates of greater than $75 \%$ among their at-risk adult patient population (Table 2). Nearly all providers performed HBsAg as part of HBV screening: $16.2 \%$ ordered HBsAg alone, $38.5 \%$ ordered both HBsAg and anti-HBs, and $40.5 \%$ ordered HBsAg, anti-HBs, and anti-HBc. Only $20.9 \%$ of providers reported vaccinating more than $75 \%$ of their eligible patients against HBV. Nearly half (43.2\%) of the providers were not familiar with AASLD guidelines.

The majority of providers surveyed were aware that chronic $\mathrm{HBV}$ infection is often asymptomatic, that $\mathrm{HBV}$ vaccination can help decrease rates of hepatocellular carcinoma, and that uninfected household contacts of patients who are HBV carriers should be vaccinated against HBV (Table 3 ). The most common factors influencing the decision to screen for HBV
Table 1. Provider and Practice Characteristics

\begin{tabular}{|c|c|}
\hline Characteristic & $\begin{array}{l}\text { All providers } \\
(N=148)\end{array}$ \\
\hline \multicolumn{2}{|l|}{ Age, years $(\mathrm{N},[\%])$} \\
\hline $20-39$ & $66(44.6)$ \\
\hline $40-59$ & $67(45.3)$ \\
\hline$\geq 60$ & $11(7.4)$ \\
\hline Not reported & $4(2.7)$ \\
\hline \multirow{2}{*}{\multicolumn{2}{|c|}{ Race/Ethnicity $(\mathrm{N},[\%])$}} \\
\hline & \\
\hline Caucasian & $87(58.8)$ \\
\hline African-American & $4(2.7)$ \\
\hline Hispanic/Latino & $10(6.8)$ \\
\hline Asian & $35(23.6)$ \\
\hline Other/Not reported & $12(8.1)$ \\
\hline U.S.-born $(\mathrm{N},[\%])$ & $123(83.1)$ \\
\hline $\begin{array}{l}\text { Providers with Asian language proficiency } \\
(\mathrm{N},[\%])\end{array}$ & $23(15.5)$ \\
\hline \multicolumn{2}{|l|}{ Post-graduate degree $(\mathrm{N},[\%])$} \\
\hline MD & $103(69.6)$ \\
\hline $\mathrm{NP} / \mathrm{PA}$ & $38(25.7)$ \\
\hline Other & $7(4.7)$ \\
\hline \multicolumn{2}{|l|}{ Specialty $(\mathrm{N},[\%])$} \\
\hline Internal medicine & $61(41.2)$ \\
\hline Family medicine & $66(44.6)$ \\
\hline Infectious diseases & $5(3.4)$ \\
\hline Other/Not reported & $16(10.8)$ \\
\hline \multicolumn{2}{|l|}{ Years in practice $(\mathrm{N},[\%])$} \\
\hline $0-10$ & $86(58.1)$ \\
\hline $11-20$ & $37(25.0)$ \\
\hline$>20$ & $22(14.9)$ \\
\hline Not reported & $3(2.0)$ \\
\hline \multicolumn{2}{|l|}{ Number of patients seen per week $(\mathrm{N},[\%])$, patients } \\
\hline $0-20 \quad$ Pa & $53(35.8)$ \\
\hline $21-40$ & $44(29.7)$ \\
\hline $41-60$ & $27(18.2)$ \\
\hline$>60$ & $20(13.5)$ \\
\hline Not reported & $4(2.7)$ \\
\hline $\begin{array}{l}\text { Provider practice consists of more than } 25 \% \text { Asian } \\
\text { patients }(\mathrm{N},[\%])\end{array}$ & $71(48.0)$ \\
\hline $\begin{array}{l}\text { Provider practice consists of more than } 50 \% \text { of } \\
\text { patients with limited English proficiency }(\mathrm{N},[\%])\end{array}$ & $59(40.0)$ \\
\hline
\end{tabular}

Table 2. HBV Screening and Vaccination Practices by Provider Self-Report

\begin{tabular}{lc}
\hline \hline HBV screening and management & $\begin{array}{c}\text { All providers } \\
(\mathbf{N}=\mathbf{1 4 8})\end{array}$ \\
\hline Proportion of adult patients screened for HBV (N, [\%]) & $18(12.2)$ \\
$1-25 \%$ & $45(30.4)$ \\
$26-50 \%$ & $35(23.6)$ \\
$51-75 \%$ & $49(33.1)$ \\
$>75 \%$ & $1(0.7)$ \\
Uncertain/ Not Reported & \\
Tests ordered for HBV screening (N, [\%]) & $24(16.2)$ \\
HBsAg & $57(38.5)$ \\
HBsAg + anti-HBs & $60(40.5)$ \\
HBsAg + anti-HBs + anti-HBc (total) & $5(3.4)$ \\
Anti-HBs + anti-HBc (total) & $1(0.7)$ \\
HBsAg + anti-HBs + HBeAg & $1(0.7)$ \\
HBsAg + anti-HBs + HBeAg + HBV DNA & \\
Proportion of vaccine-eligible adult patients vaccinated against HBV (N, \\
[\%]) & $17(11.5)$ \\
$1-25 \%$ & $38(25.7)$ \\
$26-50 \%$ & $52(35.1)$ \\
$51-75 \%$ & $31(20.9)$ \\
$>75 \%$ & $10(6.8)$ \\
Uncertain/ Not Reported & $1(0.7)$ \\
Self-rated familiarity with AASLD guidelines for management of \\
chronic HBV infection (N, [\%]) \\
Not familiar & $64(43.2)$ \\
Somewhat/Very familiar & $83(56.1)$ \\
Not reported & $1 \%$ \\
\hline
\end{tabular}


Table 3. Provider Knowledge, Attitudes, and Barriers Regarding HBV Screening and Management $(N=148)$

Provider knowledge (mean score $8.5 \pm 1.8$ )

Chronic hepatitis B is often asymptomatic. (Correct answer: Agree)

The majority of the world's population live in hepatitis B-endemic areas. (Correct answer: Agree)

Screening for liver cancer among hepatitis B patients is cost-effective. (Correct answer: Agree)

Vaccination against hepatitis $\mathrm{B}$ can prevent liver cancer. (Correct answer: Agree)

Treating hepatitis B can prevent cirrhosis (Correct answer: Agree)

Uninfected household contacts of hepatitis $\mathrm{B}$ carriers do not need to receive hepatitis $B$ vaccination. (Correct answer: Disagree)

High levels of hepatitis B viral load are associated with increased incidence of cirrhosis. (Correct answer: Agree)

In patients with hepatitis $\mathrm{B}$, liver cancer occurs only in the setting of cirrhosis. (Correct answer: Disagree)

All patients with hepatitis B should be treated. (Correct answer: Disagree)

The prevalence of chronic hepatitis B in this country remains high due to high rates of acute hepatitis B. (Correct answer: Disagree)

The balance of risks and benefits associated with liver cancer screening in patients with chronic hepatitis B is clearly known. (Correct answer: Disagree)

Provider attitude (mean score 5.5 \pm 1.7 )

What factors influence you to order screening tests?

When there is good evidence that screening leads to decreased mortality

When it is recommended by a national organization

When I see an increased frequency of that disease in my practice

When my patients ask for it

When it is used as a quality measure at my institution or by insurance companies

When it is covered by health insurance

Not screening for liver cancer among patients with hepatitis B is a malpractice risk

There are mostly other factors

Provider-perceived barriers (mean score $1.3 \pm 1.4)$

Difficulty accessing specialty (GI/hepatology) care

Language access barriers with patient

Patient financial barriers

Lack of blood testing resources

Lack of clarity of liver cancer screening guidelines

Uncertain/unaware of liver cancer screening guidelines

Discomfort with discussing liver cancer screening

Lack of effective treatment for liver cancer Other

included good evidence that screening leads to decreased mortality (96.6\%) and recommendations from a national organization $(91.9 \%)$. While $61.4 \%$ indicated that health insurance coverage influenced their decision to screen, $78.5 \%$ would order screening if it were used as a quality measure by their institution or insurance companies. The most commonly cited barriers to HBV screening were lack of clarity regarding screening guidelines $(25.0 \%)$, uncertainty or unawareness of guidelines $(23.7 \%)$, difficulty accessing specialty care $(26.4 \%)$, and patient financial barriers $(14.9 \%)$.

\section{HBV Screening and Vaccination Practices Based on Chart Review}

Records for 20,574 API patients (20,338 Asian and 236 Hawaiian/Pacific Islander) were reviewed. Patient mean age was $52( \pm 15)$ years, and 7,523 (36.6\%) were male. With respect to $\mathrm{HBV}$ testing practices, 7,926 (38.5\%) had no HBV tests, 8,978 (43.6 \%) were tested with both HBsAg and anti-HBs, and the remaining patients were tested for either HBsAg or anti-HBs alone (Fig. 1a). Among patients tested with both HBsAg and anti-HBs, $9.1 \%(821 / 8,978)$ were HBV-infected (HBsAg-positive) and $36.5 \%(3,274 / 8,978)$ were HBV-susceptible (both HBsAg and anti-HBs were negative). Appropriately, 5,511 with preexisting HBV immunity (anti-HBs positive) were not vaccinated, but only $47.4 \%$ $(1,551 / 3,274)$ of HBV-susceptible patients were vaccinated against $\mathrm{HBV}$. Of the 3,065 patients who underwent $\mathrm{HBV}$ vaccination, $50.6 \%(1,551)$ were susceptible to $\mathrm{HBV}$ infection (HBsAg-negative), but $29.7 \%$ (911) had preexisting immunity and $1.3 \%$ (40) were infected with HBV (HBsAg-positive) (Fig. 1b).

\section{Patient and Provider Factors Associated with HBV Screening}

On univariate analysis, older patient age (OR per decade 1.10, $95 \%$ CI 1.04-1.22, $p=0.004$ ), male provider sex (OR 0.97, 95 $\%$ CI $0.95-0.99, p=0.018$ ), and having a patient panel comprising over $25 \% \mathrm{HBV}$ patients (OR 0.96, $95 \%$ CI $0.93-$ $0.99, p=0.013$ ) were associated with HBV screening. Although not statistically significant, greater provider barriers (OR per unit $0.55,95 \%$ CI $0.28-1.11, p=0.094$ ) was negatively associated with HBV screening, while HBV knowledge score (OR per unit 1.28, $95 \% \mathrm{CI} 0.81-2.01, p=0.29$ ), and a favorable provider attitude (OR per unit 1.12, $95 \%$ CI 0.76-1.64, $p=0.57$ ) were positively associated with HBV screening. On multivariable analysis, HBV screening was positively associated with a favorable provider attitude score (OR per unit 1.80, $95 \%$ CI 1.18-2.74, $p=0.006$ ) and negatively associated with female patient sex (OR 0.82, $95 \%$ CI 0.73-0.92, $p=0.001$ ), higher weekly clinic patient load (OR per 20 patients $0.46,95 \%$ CI $0.28-0.76, p=0.002)$, and a higher provider barrier score (OR per unit $0.45,95 \%$ CI $0.24-0.87, p=0.017$ ) (Table 4).

To determine whether the negative association between female sex and HBV screening was age-dependent, given the recommendation of the National Perinatal Hepatitis B Prevention Program ${ }^{19}$ to test all pregnant women for $\mathrm{HBV}$ 
Table 4. Multivariable Analyses: Factors Associated with HBV Screening and Appropriate HBV Vaccination

\begin{tabular}{|c|c|c|c|c|}
\hline \multirow[t]{2}{*}{ Variable } & \multicolumn{2}{|l|}{ HBV screening* } & \multicolumn{2}{|l|}{ HBV vaccination ${ }^{\dagger}$} \\
\hline & Odds ratio $(95 \%$ CI $)$ & p value ${ }^{\S}$ & Odds ratio ${ }^{\ddagger}(95 \%$ CI $)$ & p value ${ }^{\S}$ \\
\hline Patient age (per decade) & $1.06(0.90-1.10)$ & 0.085 & $1.00(0.90-1.00)$ & 0.85 \\
\hline Patient female sex & $0.82(0.73-0.92)$ & 0.001 & $0.99(0.82-1.19)$ & 0.88 \\
\hline Provider age (per decade) & $0.67(0.29-1.50)$ & 0.33 & $0.53(0.19-1.49)$ & 0.23 \\
\hline Male provider & $1.005(0.9-1.02)$ & 0.66 & $1.00(0.99-1.02)$ & 0.65 \\
\hline Asian provider (vs. non-Asian) & $1.01(0.97-1.06)$ & 0.67 & $1.02(0.98-1.05)$ & 0.35 \\
\hline Patient load (per 20 patients) & $0.46(0.28-0.76)$ & 0.002 & $0.65(0.38-1.11)$ & 0.11 \\
\hline Provider knowledge score (per unit) & $1.42(0.84-2.39)$ & 0.19 & $1.29(0.78-2.13)$ & 0.32 \\
\hline Provider attitude score (per unit) & $1.80(1.18-2.74)$ & 0.006 & $1.29(0.91-1.85)$ & 0.16 \\
\hline Provider barrier score (per unit) & $0.45(0.24-0.87)$ & 0.017 & $0.48(0.25-0.91)$ & 0.024 \\
\hline$>25 \%$ of patient panel comprised of HBV patients & $0.96(0.91-1.02)$ & 0.22 & $0.97(0.91-1.02)$ & 0.21 \\
\hline$>50 \%$ rate of HAV vaccination among HBV patients & $0.99(0.97-1.01)$ & 0.24 & $1.01(0.99-1.03)$ & 0.59 \\
\hline$>50 \%$ rate of $\mathrm{HBV}$ vaccination & & & $1.01(0.99-1.03)$ & 0.21 \\
\hline
\end{tabular}

$* N=8,978$ for patients and $N=148$ for providers

${ }^{\dagger} N=1,551$ for patients and $N=148$ for providers

Odds ratios and confidence intervals are for each variable adjusted for all other variables in the same column.

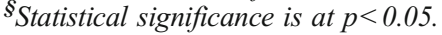

Values in bold represent statistically significant findings $(p<0.05)$

infection, we further stratified HBV screening rates by childbearing age ( $<45$ vs. $\geq 45$ years of age). The negative association between female sex and HBV screening was more pronounced in the group of childbearing age (OR 0.71, $95 \%$ CI $0.50-1.00, p=0.053)$ compared to the non-childbearingage group (OR 0.96, $95 \%$ CI 0.78-1.17, $p=0.67$ ).

\section{Patient and Provider Factors Associated with HBV Vaccination}

On univariate analysis, once again male provider sex (OR 0.97, $95 \%$ CI $0.95-0.99, p=0.003$ ), higher provider barrier score (OR per unit $0.53,95 \% \mathrm{CI} 0.33-0.85, p=0.009)$, and having a
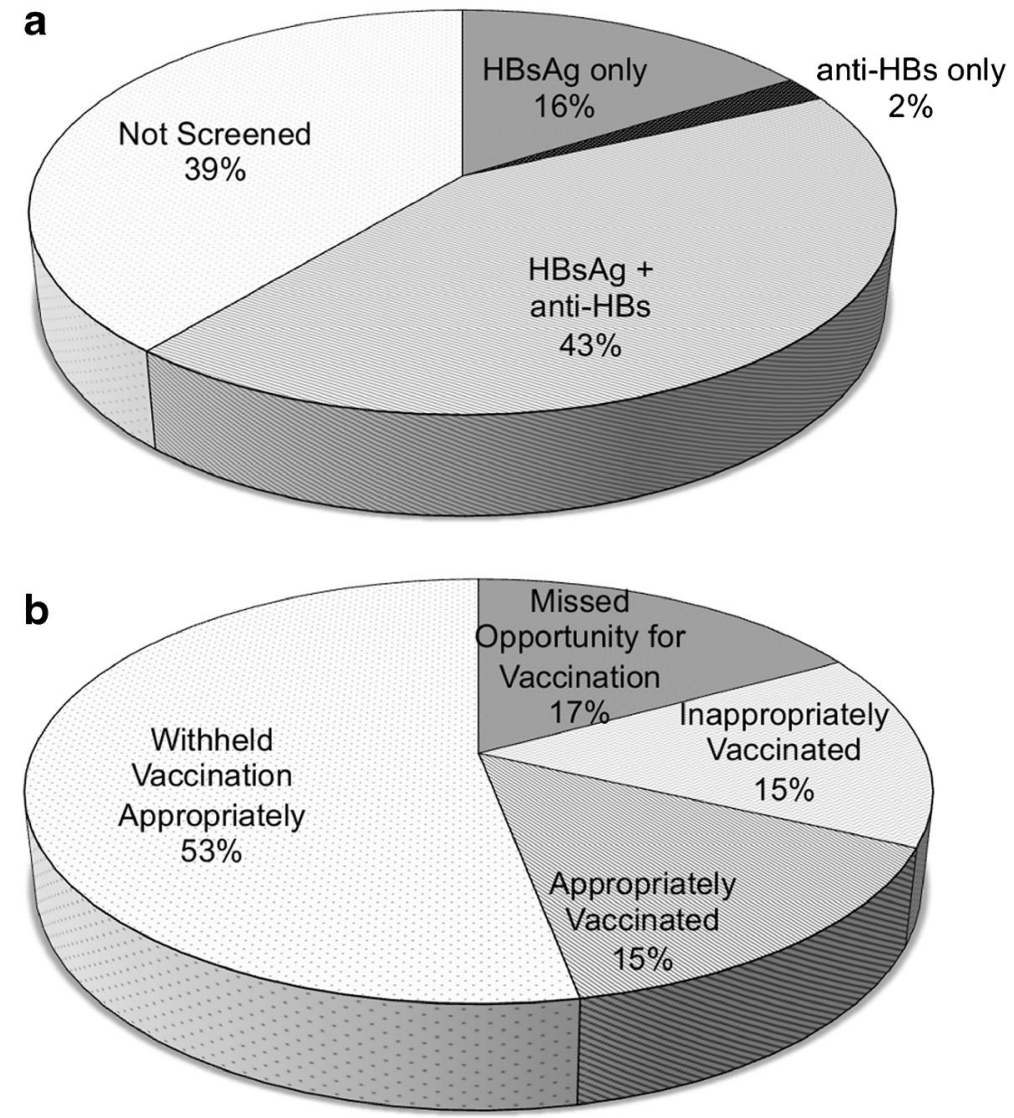

Fig. 1. HBV screening and vaccination practices based on patient record review: Panel a depicts HBV screening rates among Asian and Pacific Islanders $(N=20,574)$ and the serologic test utilized; Panel b depicts HBV vaccination practices $(N=10,299)$ based on $\mathrm{HBV}$ serology. 
patient panel comprising more than $25 \% \mathrm{HBV}$ patients (OR $0.96,95 \%$ CI $0.93-0.99, p=0.03$ ) were negatively associated with vaccination of $\mathrm{HBV}$-susceptible patients. On multivariable analysis, provider barrier score (OR per unit $0.48,95 \% \mathrm{CI} 0.25$ $0.91, p=0.024)$ was the only independent predictor of $\mathrm{HBV}$ vaccination (Table 4$)$.

\section{DISCUSSION}

To our knowledge, this is the first study to supplement provider self-report with actual clinical data to assess patient, provider, and practice characteristics associated with HBV screening and vaccination within a large urban safety-net health care system. Our data show suboptimal rates of screening and vaccination in eligible patients. Providers demonstrated a high level of knowledge of appropriate HBV preventive practices. HBV screening was associated with provider attitude and perceived barrier scores as well as patient and practice factors, while provider barrier score was the only independent predictor of $\mathrm{HBV}$ vaccination.

While CDC and AASLD guidelines recommend screening for HBV in high-risk groups, the USPSTF previously issued a " $D$ " recommendation against HBV screening in the general population, and it is unclear how this discrepancy shapes national HBV screening and vaccination practices in the primary care setting. Our study shows that providers within this healthcare system had a high overall knowledge of HBV. This is likely due to a high proportion of APIs in provider practices, as well as an intensive citywide campaign (San Francisco Hep B Free) to increase awareness of HBV in this population. ${ }^{20}$ Despite these efforts, rates of HBV screening and vaccination were suboptimal. Consistent with prior reports of HBV screening rates ranging from $35 \%$ to $65 \%,{ }^{13-17}$ our data show that only $43.6 \%$ of API patients underwent appropriate HBV screening with both HBsAg and anti-HBs. Similar to prior estimates, ${ }^{21}$ a high proportion $(9.1 \%$ ) of APIs in the safety net were HBV-infected. Although the majority of providers used appropriate HBV screening tests, less than one-third screened the majority $(>75 \%)$ of their at-risk patients. In correlation with low rates of $\mathrm{HBV}$ vaccination based on EMR review, a significant proportion of providers did not report vaccinating the majority of their eligible patients. Importantly, about one-third of vaccinated patients were already HBV-immune. These findings suggest that provider education efforts should include a focus on appropriate vaccination practices, especially in resource-limited settings.

Overall, our findings suggest that HBV screening and vaccination are primarily influenced by provider attitudes and perceived barriers to HBV care. A favorable provider attitude was positively associated with HBV screening, and providers were motivated to perform screening tests if they were evidence-based and supported by national organization recommendations. Higher provider-perceived barriers, including concerns regarding access to specialty care and patient financial barriers, were negatively associated with HBV screening and vaccination. In addition, providers who saw a higher number of patients in clinic were less apt to screen for HBV, and this likely reflects the presence of competing priorities in high-volume clinical settings. These findings suggest that provider attitudes towards screening may be enhanced by increasing their familiarity with management guidelines, and efforts to promote greater HBV screening and vaccination should focus on improving the availability and awareness of health system resources for testing, specialty referral, and treatment. This may, in turn, allow providers to better navigate competing priorities that negatively affect delivery of HBV preventive care within the primary care setting.

We identified a significant association between patient sex and HBV screening. Given the presence of a national program for prevention of vertical HBV transmission, it would be anticipated that a higher percentage of females, particularly of childbearing age, would be screened for HBV. Although most prior studies have not shown an association between patient sex and HBV screening, ${ }^{14,21,22}$ in one study, female sex was indeed associated with higher rates of HBV testing. ${ }^{23}$ In our study, however, women were less likely to be screened than men, particularly among patients less than 45 years of age. The reasons for this are unclear, but these findings may highlight the need for further evaluation of HBV screening practices among women of childbearing age in this health system. Patient English fluency and having language-concordant providers are additional factors that have been associated with higher rates of screening. ${ }^{24,25}$ In this study, however, most providers did not identify patient language as an important barrier to HBV screening, and provider Asian language proficiency was not associated with HBV screening or appropriate administration of HBV vaccination. However, the number of providers with Asian language proficiency surveyed was low $(<20 \%)$, and this may have precluded a sufficient assessment of the influence of patient-provider language concordance.

The primary limitations of the study are its retrospective design and the $45 \%$ provider response rate. Given that there was no significant difference in the proportion of provider survey responders versus non-responders from clinics with high or low HBV patient load, it is likely that survey responses are representative of the providers with variable experience in HBV care within this system. It is also well known that provider self-reporting tends to overestimate practice behavior. $^{26}$ The accompanying patient record evaluation provides additional insight into actual HBV preventive practices within this primary care setting. Patient country of birth was not available in the EMR. However, given that the mean age of API patients was 52 years, and that in approximately $60 \%$ of practices, $50 \%$ or more patients had limited English proficiency, it is likely that the majority of API patients in this safety-net population were immigrants or children of immigrants. Finally, generalizability of our findings to other clinical settings may be limited. However, given that the majority of 
individuals at risk for $\mathrm{HBV}$ are from immigrant populations with limited health care access, an understanding of the deficiencies and an improvement in HBV preventive services within a safety-net health care system that predominantly serves this population is likely to have a significant impact on reducing HBV-related health disparities.

In summary, we have confirmed suboptimal HBV screening and vaccination rates among an at-risk API population using a comprehensive approach that utilizes both provider self-report and objective patient data. Cultivating positive attitudes towards HBV care among providers by aligning and reinforcing national guidelines may help to promote improved screening and vaccination practices. Efforts to promote HBV screening and vaccination should also focus on system changes that streamline testing, specialty referral, and HBV treatment in order to overcome perceived barriers to HBV care. This study also identified an association with patient sex that supports the need for further research in order to understand potential gaps in HBV screening among API women, especially those of childbearing age. The approach utilized in this study to evaluate HBV preventive services can be applied in other healthcare settings to guide quality improvement initiatives.

Acknowledgments: Funders: This work was in part supported by San Francisco General Hospital Foundation Grant (M.K.), K24AAO22523 (M.K.), and P3O DKO26743 (UCSF Liver Center).

Conflict of Interest: The authors declare that they do not have a conflict of interest.

Corresponding Author: Mandana Khalili, MD; San Francisco General HospitalUniversity of California, San Francisco, 1001 Potrero Avenue, NH-3D, San Francisco, CA 94110, USA (e-mail: Mandana.khalili@ucsf.edu).

\section{REFERENCES}

1. Lok AS, McMahon BJ. Chronic hepatitis B: update 2009. Hepatology. 2009;50(3):661-2. doi:10.1002/hep.23190. PubMed PMID: 19714720.

2. Cohen C, Evans AA, London WT, Block J, Conti M, Block T. Underestimation of chronic hepatitis B virus infection in the United States of America. J Viral Hepat. 2008;15(1):12-3. doi:10.1111/j.1365-2893.2007.00888.x. PubMed PMID: 18088239; PubMed Central PMCID: PMC2229201.

3. Lavanchy D. Hepatitis B virus epidemiology, disease burden, treatment, and current and emerging prevention and control measures. J Viral Hepat. 2004;11(2):97-107. PubMed PMID: 14996343.

4. Mitchell AE, Colvin HM, Palmer Beasley R. Institute of Medicine recommendations for the prevention and control of hepatitis B and C. Hepatology. 2010;51(3):729-33. doi:10.1002/hep.23561. PubMed PMID: 20186842.

5. Weinbaum CM, Mast EE, Ward JW. Recommendations for identification and public health management of persons with chronic hepatitis B virus infection. Hepatology. 2009;49(5 Suppl):S35-44. doi:10.1002/hep.22882. PubMed PMID: 19399812.

6. U.S. Preventive Services Task Force. Screening for hepatitis B virus infection in nonpregnant adolescents and adults: draft recommendation statement. AHRQ Publication No. 12-05172-EF-2. Accessed May 1, 2014. Available at: http://www.uspreventiveservicestaskforce.org/uspstf12/ hepb/hepbdraftrec.htm
7. Lin SY, Chang ET, So SK. Why we should routinely screen Asian American adults for hepatitis B: a cross-sectional study of Asians in California. Hepatology. 2007;46(4):1034-40. doi:10.1002/hep.21784. PubMed PMID: 17654490

8. Bindman AB, Chen A, Fraser JS, Yee HF Jr, Ofman D. Healthcare reform with a safety net: lessons from San Francisco. Am J Manage Care. 2009; 15(10):747-50. PubMed PMID: 19845426.

9. Bloom BS, Hillman AL, Fendrick AM, Schwartz JS. A reappraisal of hepatitis B virus vaccination strategies using cost-effectiveness analysis. Ann Intern Med. 1993;118(4):298-306. PubMed PMID: 8420448.

10. Eckman MH, Kaiser TE, Sherman KE. The cost-effectiveness of screening for chronic hepatitis B infection in the United States. Clin Infect Dis Off Publ Infect Dis Soc Am. 2011;52(11):1294-306. doi:10.1093/cid/cir199. PubMed PMID: 21540206 ; PubMed Central PMCID: PMC3097367.

11. Hutton DW, Tan D, So SK, Brandeau ML. Cost-effectiveness of screening and vaccinating Asian and Pacific Islander adults for hepatitis B. Ann Intern Med. 2007;147(7):460-9. PubMed PMID: 17909207.

12. Veldhuijzen IK, Toy M, Hahne SJ, et al. Screening and early treatment of migrants for chronic hepatitis B virus infection is cost-effective. Gastroenterology. 2010;138(2):522-30. doi:10.1053/j.gastro.2009.10.039. PubMed PMID: 19879275.

13. Khalili M, Guy J, Yu A, et al. Hepatitis B and hepatocellular carcinoma screening among Asian Americans: survey of safety net healthcare providers. Dig Dis Sci. 2011;56(5):1516-23. doi:10.1007/s10620-010-1439-3. PubMed PMID: 21046247; PubMed Central PMCID: PMC3082048.

14. Coronado GD, Taylor VM, Tu SP, et al. Correlates of hepatitis B testing among Chinese Americans. J Community Health. 2007;32(6):379-90. doi:10.1007/s10900-007-9060-x. PubMed PMID: 17940869.

15. Ma GX, Shive SE, Wang MQ, Tan Y. Cancer screening behaviors and barriers in Asian Americans. Am J Health Behav. 2009;33(6):650-60. PubMed PMID: 19320614.

16. Thompson MJ, Taylor VM, Jackson JC, et al. Hepatitis B knowledge and practices among Chinese American women in Seattle, Washington. J Cancer Educ Off J Am Assoc Cancer Educ. 2002;17(4):222-6. doi:10.1080/08858190209528842. PubMed PMID: 12556060.

17. Lai CJ, Nguyen TT, Hwang J, Stewart SL, Kwan A, McPhee SJ. Provider knowledge and practice regarding hepatitis B screening in Chinese-speaking patients. J Cancer Educ Off J Am Assoc Cancer Educ. 2007;22(1):37-41. doi:10.1080/08858190701348083. PubMed PMID: 17570807.

18. Montano DE, Phillips WR. Cancer screening by primary care physicians: a comparison of rates obtained from physician self-report, patient survey, and chart audit. Am J Public Health. 1995;85(6):795-800. PubMed PMID: 7762712; PubMed Central PMCID: PMC1615496.

19. Smith EA, Jacques-Carroll L, Walker TY, Sirotkin B, Murphy TV. The national Perinatal Hepatitis B Prevention Program, 1994-2008. Pediatrics. 2012;129(4):609-16. doi:10.1542/peds.2011-2866. PubMed PMID: 22451702

20. Bailey MB, Shiau R, Zola J, et al. San Francisco hep B free: a grassroots community coalition to prevent hepatitis B and liver cancer. J Community Health. 2011;36(4):538-51. doi:10.1007/s10900-010-9339-1. PubMed PMID: 21125320; PubMed Central PMCID: PMC3130910.

21. Hur $\mathbf{K}$, Wong $\mathbf{M}$, Lee $\mathbf{J}$, Lee $\mathbf{J}$, Juon HS. Hepatitis B infection in the Asian and Latino communities of Alameda County, California. J Community Health. 2012;37(5):1119-26. doi:10.1007/s 10900-012-9553-0. PubMed PMID: 22362166.

22. Nguyen TT, McPhee SJ, Stewart S, et al. Factors associated with hepatitis B testing among Vietnamese Americans. J Gen Intern Med. 2010;25(7):694-700. doi:10.1007/s11606-010-1285-1. PubMed PMID: 20306150; PubMed Central PMCID: PMC2881980.

23. Spradling PR, Rupp L, Moorman AC, et al. Hepatitis B and C virus infection among 1.2 million persons with access to care: factors associated with testing and infection prevalence. Clin Infect Dis Off Publ Infect Dis Soc Am. 2012;55(8):1047-55. doi:10.1093/cid/cis616. PubMed PMID: 22875876.

24. Ngo-Metzger Q, Sorkin DH, Phillips RS, et al. Providing high-quality care for limited English proficient patients: the importance of language concordance and interpreter use. J Gen Intern Med. 2007;22(Suppl 2):324-30. doi:10.1007/s11606-007-0340-z. PubMed PMID: 17957419; PubMed Central PMCID: PMC2078537.

25. Thompson MJ, Taylor VM, Yasui Y, et al. Hepatitis B knowledge and practices among Chinese Canadian women in Vancouver, British Columbia. Can J Publ Health. 2003;94(4):281-6. PubMed PMID: 12873087.

26. Rauscher GH, Johnson TP, Cho YI, Walk JA. Accuracy of self-reported cancer-screening histories: a meta-analysis. Cancer Epidemiol biomarkers Prev Publ Am Assoc Cancer Res. 2008;17(4):748-57. doi:10.1158/10559965.EPI-07-2629. PubMed PMID: 18381468. 